\title{
Learning from Ideal Edge for Image Restoration
}

\author{
Jin-Ping $\mathrm{HE}^{\dagger \mathrm{a})}$, Member, Kun GAO ${ }^{\dagger \dagger}$, Guo-Qiang $\mathrm{NI}^{\dagger \dagger}$, Guang-Da $\mathrm{SU}^{\dagger \dagger \dagger}$, \\ and Jian-Sheng CHEN ${ }^{\dagger \dagger}$, Nonmembers
}

\begin{abstract}
SUMMARY Considering the real existent fact of the ideal edge and the learning style of image analogy without reference parameters, a blind image recovery algorithm using a self-adaptive learning method is proposed in this paper. We show that a specific local image patch with degradation characteristic can be utilized for restoring the whole image. In the training process, a clear counterpart of the local image patch is constructed based on the ideal edge assumption so that identification of the Point Spread Function is no longer needed. Experiments demonstrate the effectiveness of the proposed method on remote sensing images.

key words: learning-based, image restoration, ideal edge, image analogy
\end{abstract}

\section{Introduction}

A conventional solution of single-frame image restoration is to estimate the Point Spread Function (PSF) and image noises and then remove them [1]. However, the characteristics of practical imaging systems and imaging conditions vary a lot, and the degradation process is complicated and diverse [2], [3], leading to an unknown image degradation. In practice, therefore, it is hard to accurately estimate the PSF [4], [5]. Facing this problem, we propose a novel learning-based algorithm in which precise estimation of the PSF is not necessary.

The learning-based algorithm for resolution enhancement was first proposed by Freeman et al. in [6]. Baker et al. [7], [8] applied a learning-based technique, called the hallucinating faces, to facial images. In the hallucinating faces algorithm, the training images are restricted to a small subset containing similar face images. Hertzmann et al. [9] proposed an algorithm using the concept of image analogy in the super-resolution restoration of color images. Nevertheless, both Baker's method and Hertzmann's method rely heavily on the selection of training samples, of which the applicability and reliability may be dubious. This will essentially affect the reproducibility and consistency of the recovering process. In this paper, we propose a learning-based image restoration method, in which one local image patch is retrieved from the image to be restored. A clear counterpart

\footnotetext{
Manuscript received November 30, 2012.

Manuscript revised July 6, 2013.

The author is with Beijing Institute of Space Mechanics \& Electricity, Beijing 100190, China.

${ }^{\dagger \dagger}$ The authors are with the School of Optoelectronics, Beijing Institute of Technology, Beijing 100081, China.

${ }^{\dagger \dagger}$ The authors are with the Department of Electronics Engineering, Tsinghua University, Beijing 100084, China.

a) E-mail: hjping@mail.tsinghua.edu.cn

DOI: 10.1587/transinf.E96.D.2487
}

of this image patch is then constructed according to the ideal edge assumption. The local image patch and its clear counterpart are used as the training sample pair in the restoration process. Clearly, the selection of the local image patch and the construction of its clear counterpart are crucial to our method.

Edges in images are believed to contain abundant information. They universally have small gradients because of sampling and degradation. The edge transition areas are located in those gradient areas. Natural images can usually be divided into flat areas and edge transition zones. Image blurring is basically caused by the superposition of neighboring pixels. Therefore, the effect of blurring is not obvious in flat image areas in which neighboring pixels usually have similar pixel values. The blurring effect is perceived mostly in edge transition zones, which provides key clues for restoring blurred images. So the blurred training image patch should contain an edge transition zone at least.

The construction of the clear counterpart of the selected training patch is performed according to the following observation: boundaries of objects are often ideal in reality. These real world ideal edges are usually blurred during the image acquisition process. Therefore, an ideal edge image patch can be regarded as a fair guess of the unknown clear counterpart of the selected training patch.

The rest of this paper is organized as follows. Section 2 introduces our image restoration algorithm. It mainly consists of four parts: a general description of our algorithm; the selection of the local image patch and the construction of its clear counterpart, namely the ideal edge image patch; pixel position detection; and adaptive pixel compensation. Section 3 presents experimental results, and Sect. 4 concludes our work.

\section{Image Restoration Algorithm}

\subsection{Algorithm Overview}

The overall scheme of our proposed method is shown in Fig. 1, in which $I_{b}$ is the blurred image to be restored. In Fig. $1, I_{a}$ is an image patch selected from image $I_{b}$ containing edge transition zones; and $I_{a a}$ is the ideal edge image patch constructed from $I_{a}$. $I_{a}$ and $I_{a a}$ are used as the training sample pair in the image restoration. Each pixel of $I_{b}$ is firstly categorized as of belonging to flat areas or edge transition zones according to the resemblance to $I_{a}$ pixels. Then, 


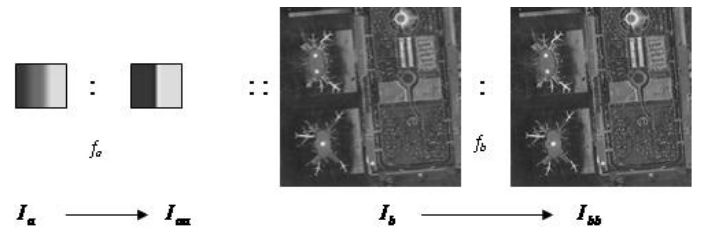

Fig. 1 An illustration of the proposed algorithm.

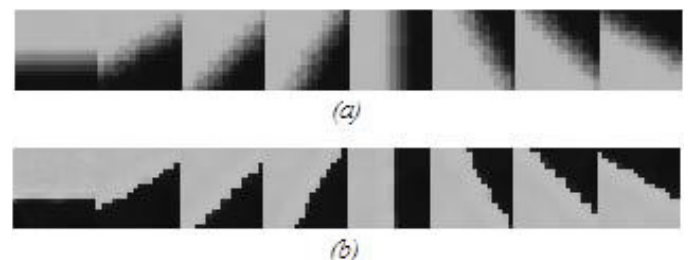

Fig. 2 The edge images with different directions: (a) the blurred edge images generated by the defocusing degradation model with radius $=3$; (b) the ideal edges corresponding to (a).

according to the position information, the restored image $I_{b b}$ is generated by modifying pixel values of $I_{b}$ using adaptive pixel compensation, which is designed according to the pixel value transition rule learned from the training sample pair.

In our restoration algorithm, images $I_{a}, I_{a a}$ and $I_{b}$ are the inputs, and image $I_{b b}$ is the output restored image. From the image analogy point of view, $I_{b b}$ is related to $I_{b}$ in the same way that $I_{a a}$ relates to $I_{a}$. For better understanding, we adopt the notation of image analogy [9] although our algorithm differs substantially from that of Hertzmann in terms of both underlying rationale and implementation details. Let $f_{a}$ denotes the mapping from image $I_{a}$ to $I_{a a}$ (it can be represented by $I_{a} \rightarrow I_{a a}$ ), and $f_{b}$ denotes the mapping of $I_{b} \rightarrow I_{b b}$, then " $f_{a}:: f_{b}$ " represents that $f_{a}$ is identical to $f_{b}$.

\subsection{Training Sample Pair Acquisition}

As we have stated before, the local image patch for training will be selected with containing edge transition zones of $I_{b}$. Nevertheless, directly cropping local image patches containing blurred edge from $I_{b}$ may bring about difficulties in ideal edge image patch construction. Figure 2 shows such an example. Due to the variation and inaccuracy in digitizing image edges, non-horizontal or non-vertical edges shown in Fig. 2 will introduce extra distortion and noises.

To solve this problem, we adopt the horizontal or vertical edge images as training pair. The detail operation is selecting one point in edge transition fields from image $I_{b}$ (You can select the point several times until obtaining the optimal view results) and getting the line section along the edge normal direction through that point. A square image $I_{a}$ is constructed through making each row vector or column vector of image $I_{a}$ equal to the line section (see Fig. 3). Of course, we can directly select a square patch containing horizontal or vertical edges as shown in Fig. 3, if strict horizontal or vertical edge patches exist. The sample image $I_{a}$

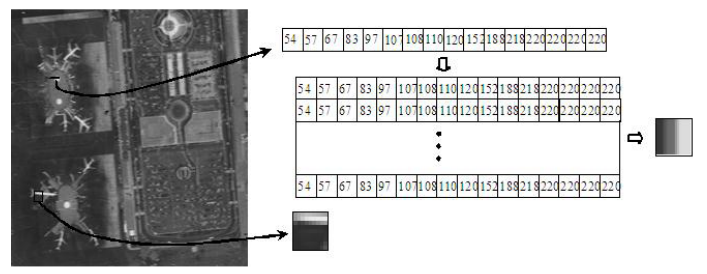

Fig. 3 An illustration of the blurred sample construction or selection.
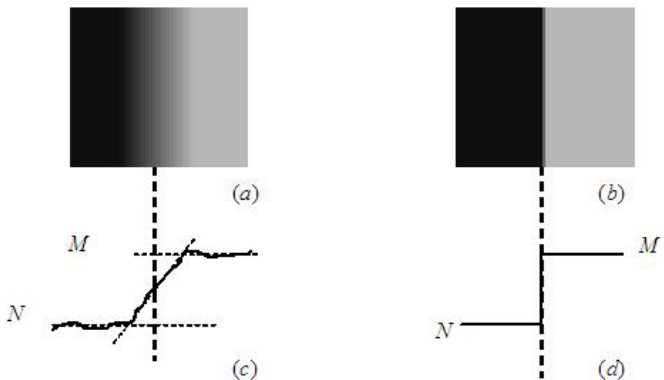

Fig. 4 An illustration of blurred and ideal edges: (a) A blurred edge image $I_{a}$; (b) An ideal edge image $I_{a a}$; (c) The cross-section of $I_{a}$; (d) The cross-section of $I_{a a}$.

is an appearance of blurred characteristics for image $I_{b}$.

Real image edges are very complex shown in Fig. 4 (a). They universally have small gradients (see Fig. 4 (c)). Those areas are named as edge transition zones by us. Comparing with real image edges, an ideal edge has a strict and clear boundary [10] without edge transition zone (see Fig. 4 (b)). Its cross-section can be considered as a step function (see Fig. 4(d)). Based on the analysis of Sect. 1, ideal edge objectively exists. And an ideal edge patch can be regarded as a fair guess of the unknown clear counterpart of the above blurred patch.

The grayscale values of two flat areas in image $I_{a}$ respectively have unpredictable ups and downs around averages $M$ and $N(M>N)$ (see Fig. 4(c)). Constructing the ideal edge $I_{a a}$ is through making the image $I_{a}$ binary with the two averages $M$ and $N$. The threshold of side boundary is $(M+N) / 2$. We can obtain each point value $I_{a a}(i, j)$ as follows:

$$
I_{a a}(i, j)=\left\{\begin{array}{cc}
M & I_{a}(i, j)>(M+N) / 2 \\
N & I_{a}(i, j) \leq(M+N) / 2
\end{array}\right.
$$

The threshold setting is based on the analysis of edges on both sides convolving with a symmetric blurred window [4].

Comparing the contents of $I_{a}$ and $I_{a a}$, we just utilize a simple and reasonable operation to remove the edge transition zone in order to eliminate blur. Obviously, the blurred images with a large frame or containing complicated textures are difficult to be reconstructed through the above direct method (see (1)). So we can learn the manner of removing edge transition zone from $I_{a}$ to $I_{a a}$ to restore complicated blurred images. 


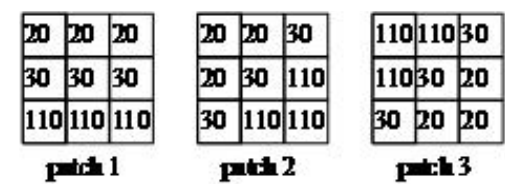

Fig. 5 Center points with same $D D$ value in different patches.

\subsection{Pixel Position Detection}

Hertzmann et. al [9] firstly searched the most matching point $(p, q)$ in image $I_{a}$ for one point $(i, j)$ in image $I_{b}$, using the weighted distance over the feature vectors after the transformation of Gaussian Pyramid. Compared with Hertzmann's, the content of our training sample $I_{a}$ is more simplex. Image $I_{a}$ just consists of two flat areas and one edge transition zone. In order to remove the edge transition zones of $I_{b}$, the first task in our algorithm is categorizing each pixel of $I_{b}$ as of belonging to flat areas or edge transition zones, referring to image $I_{a}$. While the information of $I_{a}$ for reference is quite limited. So we need to design a new transform domain based on relative value instead of absolute value in order to increase the comparability of $I_{a}$.

In the new proposed transform domain, the characteristic matrix $D D_{a}$ corresponding to image $I_{a}$ is calculated through (2) and (3).

$$
\begin{aligned}
& a v_{a}(i, j, R)=\frac{1}{(2 R+1)^{2}} \times \sum_{k=-R, l=-R}^{R, R} I_{a}(i+k, j+l) \\
& D D_{a}(i, j)=\sum_{k=-R, l=-R}^{R, R}\left\|I_{a}(i+k, j+l)-a v_{a}(i, j, R)\right\|^{2}
\end{aligned}
$$

The value $R$ can be $1,2,3$, and so on, and $2 R+1$ denotes the width of local moving window. For example, when $R=2$, the value of $D D_{a}(i, j)$ for point $(i, j)$ represents fluctuating condition around the $5 \times 5$ neighborhood average. The value of $D D_{a}(i, j)$ is a statistic which can express the local texture characteristic. We describe the novel transform domain in terms of the "Local Relative Transform Domain (LRTD)".

When one point is at the flat areas, it has a little fluctuation around window average. So its $D D$ value is smaller than the points' at the edge transition zones. All the $D D$ values in flat areas are similar and converge to zero. Further more, it is designed as non-negative parameter, so it is nondirectional. Shown in Fig. 5, the center points of the three patches have the same $D D$ values, so they have the same position. If patch 1 in Fig. 5 which is horizontal belongs to image $I_{a}$ and patch 2, 3 which are diagonal belong to image $I_{b}$, through comparing the center point of patch 1 , the center points of patch 2, 3 can be found out and their position information are definite. Although our training samples are horizontal or vertical, the non-horizontal and non-vertical edges can be equally detected out in LRTD.

Based the above analysis, the similarity of $D D$ values in different images can be directly utilized to identify pixel position. The most matching point in image $I_{a}$ for one point $(i, j)$ in image $I_{b}$ is searched by using (4).

$$
(p, q)=\arg \min _{(m, n)}\left\|D D_{a}(m, n)-D D_{b}(i, j)\right\|^{2}
$$

\subsection{Adaptive Pixel Compensation}

After finding the most matching point $(p, q)$, Hertzmann et. al just let the value of $(p, q)$ equal to the value of $(i, j)$ in the transformation field of Gaussian Pyramid. Different from the Hertzmann's method, the matching point was found in LRTD. We incline to compensate the $I_{b b}(i, j)$ value to be coherent with the absolute gray value conditions of $I_{b}(i, j)$, rather than directly make $I_{a a}(p, q)$ equal to $I_{b b}(i, j)$. According to the above construction of LRTD, we implement adaptive pixel compensation based on the relative amplitude in the neighborhood.

The difference of $I_{b b}(i, j)$ and $I_{a a}(p, q)$ can be measured by the relative amplitude in the neighborhood as shown in (2) and (5). The mapping of $I_{a} \rightarrow I_{a a}$ can be thought of the amplitude ratio as shown in (6). If suppose image $I_{b b}$ has the same type with image $I_{a a}, I_{a} \rightarrow I_{a a}$ and $I_{b} \rightarrow I_{b b}$ have the same mapping as (7). The adaptive compensation (8) can be obtained based on (5), (6) and (7).

$$
\begin{aligned}
& S_{a}(p, q)=I_{a}(p, q)-a v_{a}(p, q, R) \\
& f_{a}(p, q)=\frac{S_{a}(p, q)}{S_{a a}(p, q)} \\
& f_{a}(p, q)=f_{b}(i, j) \\
& \frac{I_{a}(p, q)-a v_{a}(p, q, R)}{I_{a a}(p, q)-a v_{a a}(p, q, R)}=\frac{I_{b}(i, j)-a v_{b}(i, j, R)}{I_{b b}(i, j)-a v_{b b}(i, j, R)}
\end{aligned}
$$

Suppose $I_{b}$ and $I_{b b}$ have the same neighbor mean shown in (9). Then we can obtain the value $I_{b b}(i, j)$ according to (8) and (9) shown in (10).

$$
\begin{aligned}
& a v_{b b}(i, j, R)=a v_{b}(i, j, R) \\
& I_{b b}(i, j)=a v_{b}(i, j, R)+S_{b}(i, j) \times S_{a a}(p, q) / S_{a}(p, q)(10)
\end{aligned}
$$

Especially, when the matching point $(p, q)$ is located in the strict flat areas $\left(S_{a}(p, q)=0\right)$, we demand $f_{a}(p, q)=1$. Then we simplify $(10)$ and get $I_{b b}(i, j)=I_{b}(i, j)$. So the pixel compensation just modifies the pixels in edge transition zones of $I_{b}$. It is adaptive for different areas. To sum up the above arguments, the final compensation formula is shown in (11).

$$
I_{b b}(i, j)=\left\{\begin{array}{cc}
a v_{b}(i, j, R)+\frac{S_{a a}(i, j) \times S_{a a}(p, q)}{S_{a}(p, q)} & \text { if } S_{a}(p, q) \neq 0 \\
I_{b}(i, j) & \text { if } S_{a}(p, q)=0
\end{array}\right.
$$

In a word, the input of our proposed algorithm is a blurred image $I_{b}$. The first tasks are selecting blurred sample $I_{a}$ and constructing ideal edge image $I_{a a}$. Then for one point $(i, j)$ of image $I_{b}$, we get the optimal matching point $(p, q)$ through (4). Finally, the value of $I_{b b}(i, j)$ is obtained by implementing adaptive pixel compensation according to 


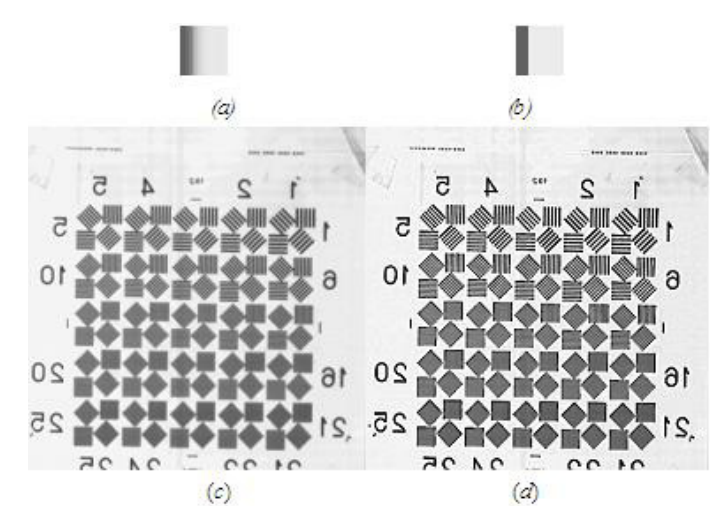

Fig. 6 The recovery result learning from ideal edge: (a) The blurred sample image selected from image (c); (b) The ideal edge image constructed from image (a); (c) The blurred image of WT1005-62 resolution panel; (d) The recovery image learning from ideal edge.

Table 1 The statistical data of the subjective visual evaluation.

\begin{tabular}{crrrrrrr}
\hline $\begin{array}{c}\text { The increased number of identified } \\
\text { groups after restoration }\end{array}$ & -2 & -1 & 0 & 1 & 2 & 3 & 4 \\
\hline The number of people & 0 & 0 & 1 & 25 & 23 & 1 & 0 \\
\hline
\end{tabular}

(11). Repeat the above steps until processing all points in image $I_{b}$.

\section{Experimental Results}

In the experiments, the value of $R$ is 2 . The proposed algorithm is firstly tested on the blurred image of WT100562 resolution panel for subjective assessment. The size of Fig. 6 (a) and Fig. 6 (b) is $16 \times 16$. And they comprise the training sample pair. The Fig. 6 (c) learns the mapping from this pair. The recovery result of WT1005-62 resolution panel (see Fig. 6(d)) enhanced one group of line pairs at least through subjective visual evaluation of fifty people (see Table 1).

Through visual quality comparison between the original image (see Fig. 6(c)) and the recovery image (see Fig. 6 (d)), the recovery image obviously becomes clear.

Secondly, we use image contrast ratio and image entropy as the objective assessment. The image contrast ratio shown in (12) is the average of all local contrast ratios in neighborhoods. $a v(\bullet)$ denotes the average operator. $c_{i j}$ denotes the pixel set of $5 \times 5$ neighborhood centered by point $(i, j)$. $\max (\bullet)$ denotes the maximal operator and $\min (\bullet)$ denotes the minimal operator.

$$
C=\operatorname{av}\left(\sum_{i} \sum_{j} \frac{\max \left(c_{i j}\right)-\min \left(c_{i j}\right)}{\max \left(c_{i j}\right)+\min \left(c_{i j}\right)}\right)
$$

In formula (13), $P_{i}$ is the probability of occurrence for gray value $i$ in image $I$ and $L$ is the total number of gray levels. The image entropy is defined as follows:

$$
H(I)=-\sum_{i=0}^{L-1} P_{i} \log _{2}\left(P_{i}\right)
$$

Table 2 The comparison of objective assessments for recovery.

\begin{tabular}{lrr}
\hline & Image contrast ratio $C$ & Image entropy $H$ \\
\hline Fig. 6 (c) & 0.11577 & 5.2923 \\
Fig. 6(d) & 0.36705 & 6.1068 \\
Fig. 7 (a) & 0.20858 & 6.4753 \\
Fig. 7 (b) & 0.53427 & 6.8131 \\
Fig. 7 (c) & 0.40710 & 6.8173 \\
\hline
\end{tabular}
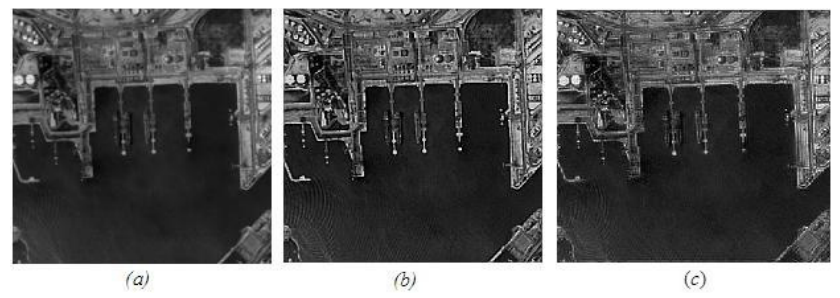

Fig. 7 The recovery result of a remote sensing image containing blurred water wave: (a) The original image; (b) The recovery image learning from ideal edge; (c) The blind recovery result with maximum likelihood.

The bigger the values are, the better the image qualities are. From Table 2, we can get that the definition of the recovery image is better than pretreatment's. And shown on the lower left corner of recovery image (see Fig. 7 (b)), the water wave is clearly presented. While it is almost invisible in the blurred image (see Fig. 7 (a)). Although the water wave has also been restored in the recovery result of maximum likelihood algorithm, there are seriously ringing effects nearby the edge positions. The ringing effects would take the false information and cause the misunderstanding. The entropy value of Fig. 7 (c) is bigger than Fig. 7 (b)'s because of those ringing effects. In a certain sense, the output image of our algorithm is a synthesized image. However, the recovery content could supply credible and available information, because of the real existent fact of ideal edge.

\section{Conclusions}

This paper presents a new recovery algorithm that uses selflearning. Our basic idea is to remove edge transition zones to eliminate blur, because blurring always appears in edge transition fields. Based on this understanding, sample pairs containing edges are constructed. The characteristic matrix and the relevant compensation are also the innovation points of our method. Learning from ideal edges makes the restoration results reasonable, and the results are obviously clear.

\section{Acknowledgments}

The work is supported by the 973 Foundation of China (613210).

\section{References}

[1] R.L. Lagendijk and J. Biemond, "Block-adaptive image identification and restoration," Proc. ICASSP, vol.4, pp.2497-2500, 1991.

[2] H. Kaufman and A.M. Tekalp, "Survey of estimation techniques in image restoration," IEEE Control Syst. Mag., vol.11, pp.16-24, 
1991.

[3] M.J. Wahoske, R.-W. Liu and R.L. Stevenson, "Dual-receiver blind identification for image blurs," IEEE International Symposium on Circuits and Systems, vol.2, pp.1377-1380, 1997.

[4] J. Biemond, F.G. Van der Putten, and J.W. Woods, "Identification and restoration of images with symmetric noncausal blurs," IEEE Trans. Circuits Syst., vol.358, pp.385-393, 198.

[5] M.-Y. Zou, "Deconvolution and Signal Recovery," National Defence Industry Press., Beijing, 2001.

[6] W.T. Freeman, E.C. Pasztor, and O.T. Carnichael, "Learning lowlevel vision,” Int. J. Comput. Vis., vol.40, no.1, pp.25-47, 2000.
[7] S. Baker and T. Kanade, Hallucinating faces, The Robotics Institute, Pennsylvania, Pittsburg, Carnegie Mellon University Press, 1999.

[8] S. Baker and T. Kanade, "Limits on super-resolution and how to break them," IEEE Conf on Computer Vision and Pattern Recognition, vol.2, pp.372-379, 2000.

[9] A. Hertzmann, C.E. Jacobs, N. Oliver, B. Curless, and D.H. Salesin, "Image anologies," Proc. 28th Annual Conf. on Computer Graphics and Interactive Techniques SIGGRAPH, pp.327-340, 2001.

[10] Y.-T. Gu and E.-H. Wu, "Image-analogies based super resolution," J. Software, vol.19, no.4, pp.851-860, 2008. 